\section{Non-planar beam-wall joints in tall building structures}

\author{
A. K. H. Kwan, BSc(Eng), PhD, CEng, MICE and W. T. Chan, BEng, MSc(Eng), \\ MIStructE
}

Stress concentration and local deformation occur at both coplanar and non-planar beam-wall joints. However, over the years, only coplanar joints have been studied in detail. Very few studies on nonplanar joints have been carried out and most designers still treat non-planar joints as hinges and neglect the possible coupling effect of beams connected to such joints. There is also no established method for detailed design of non-planar joints. Herein, a parametric study of the behaviour of non-planar beam-wall joints using finite-element analysis is described. On the basis of numerical results, a simple equivalent-frame model is developed. Examples are given to illustrate its applications and the results show that the coupling effect of beams connected to nonplanar joints can be very substantial. Moreover, in order to avoid cracking at the joints, it is proposed that in the detailed design (a) the concentrated out-of-plane moment acting from the beam on the wall should be catered for by designing a vertical strip of the wall, having a width as determined in the present study, as a column to resist such a moment; and (b) the bending moment induced in the beam should be properly allowed for and the longitudinal reinforcement so provided adequately anchored into the wall.

Keywords: beams \& girders

\begin{tabular}{ll}
\multicolumn{2}{l}{ Notation } \\
$B$ & breadth of beam \\
$B_{\mathrm{b}}$ & effective depth of fictitious beam \\
$B_{\mathrm{c}}$ & effective width of fictitious column \\
$D$ & depth of beam \\
$E$ & modulus of elasticity \\
$G$ & shear modulus \\
$H$ & height of storey \\
$I$ & moment of inertia of fictitious column \\
$J$ & torsional inertia of fictitious beam \\
$K_{\theta}$ & rotational stiffness of beam-wall joint \\
$L$ & length of beam from joint to point of \\
& contraflexure \\
$T$ & thickness of wall \\
$W$ & width of wall \\
$v$ & Poisson's ratio
\end{tabular}

\section{Introduction}

Shear walls and core walls are the most commonly used structural forms for tall concrete buildings. They have high structural efficiency and, apart from the structural purpose of providing lateral stiffness and strength, also serve the architectural purpose of partition/external walls or utility shafts. Their structural efficiency is often further increased by connecting individual shear/core walls together through coupling beams so that in effect a coupled shear/core wall system is formed. Depending on the layout of the building, the coupling beams may be coplanar with the walls that they are connected to, as shown in Fig. 1, or fall within a plane that is not coplanar with the walls, as shown in Fig. 2.

2. The coupling effect of coplanar coupling beams, i.e. coupling beams which fall within the same plane as the wall panels that they are connected to, has been quite thoroughly investigated by many researchers. ${ }^{1-3}$ Under favourable conditions, coplanar coupling beams can increase the lateral stiffness of the structural system by more than $100 \%$. However, the coupling effect of the beams is often significantly reduced by the local deformation at the beam-wall joints which arises as a result of stress concentration there. Stress concentration and local deformation at coplanar beam-wall joints have been studied in detail by Michael, ${ }^{4}$ Bhatt ${ }^{5}$ and Kwan. ${ }^{6}$ Roughly speaking, the local deformation at a coplanar beam-wall joint is equivalent to a slight extension of the beam end into the wall by an amount equal to approximately one-quarter to one-half of the beam depth. Despite the reduction in effective beam stiffness by the local deformation at the beam-wall joints, coplanar coupling beams can still substantially increase the structural efficiency of a shear/core wall system.

3. The situation with non-planar coupling beams, i.e. coupling beams which do not fall within the same plane as the wall panels that they are connected to, is somewhat more complicated because the non-planar coupling beams would induce out-of-plane bending moments in the walls near the beam-wall joints. Owing to the relatively small thickness of the walls compared with the width, the outof-plane bending stiffness of the walls is
Proc. Instn Civ. Engrs Structs \& Bldgs, 2000, 140, Feb., 73-83

Paper 12104

Written discussion closes 31 August 2000

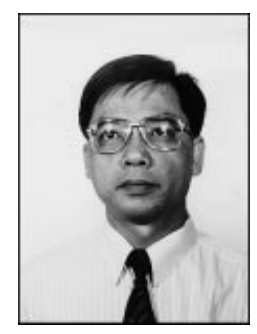

A. K. H. Kwan, Senior Lecturer, Department of Civil Engineering, University of Hong Kong

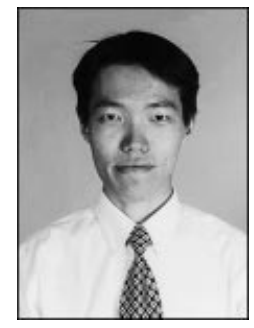

W. T. Chan

Engineer, Ove Arup \& Partners H.K. Ltd. 
usually neglected and the walls are simply modelled by plane stress elements in the analysis. With the out-of-plane bending stiffness neglected, the non-planar beam-wall joints can only be treated as hinges and consequently the coupling effect of the non-planar coupling beams has to be discarded. Owing to the assumption that the non-planar beam-wall joints act like hinges, the non-planar coupling beams are often designed as simply supported beams and the possible out-of-plane bending of the walls is ignored during reinforcement detailing. While treating the non-planar joints as hinges would tend to underestimate the lateral stiffness and strength of the structural system and hence should be on the conservative side from the point of view of the overall structural performance, neglect of the possible bending of the beams and walls near the joints in reinforcement detailing may lead to serious cracking of the concrete near the joints.

However, at present, there is no established method of design giving guidance on how to provide reinforcement to cater for the bending stresses around the joints.

4. Very few studies on the coupling effect of non-planar coupling beams have been carried out. So far, the only studies conducted on this topic are those due to Roberts and his coworkers, ${ }^{7,8}$ who have developed a theoretical model for analysing the structural behaviour of core walls partially closed by non-planar coupling beams similar to the one shown in Fig. 2(a). In the theoretical model, the coupling effect of the non-planar coupling beams is taken into account by considering the out-ofplane bending stiffness of the walls and treating the band of non-planar coupling beams as an equivalent shear diaphragm. Experiments have also been performed to verify the theoretical predictions. Both the theoretical and the experimental results revealed that the coupling effect of the non-planar coupling beams can substantially increase the torsional stiffness of a core wall and that the out-of-plane bending of the side walls has significant influence on the effectiveness of the non-planar coupling beams. However, this method of treating the non-planar coupling beams as an equivalent shear diaphragm is based on an assumed out of-plane bending shape of the walls which is yet to be justified. In other words, the out-ofplane bending of the walls is not evaluated as part of the solution taking into account the stress concentration at the joints. Generally, the use of an assumed displacement mode instead of the actual one evaluated as an integral part of the solution would lead to an overestimation of the stiffness. Moreover, this method is geared more for hand calculation of relatively simple core wall structures than for computer analysis of more general structural forms.

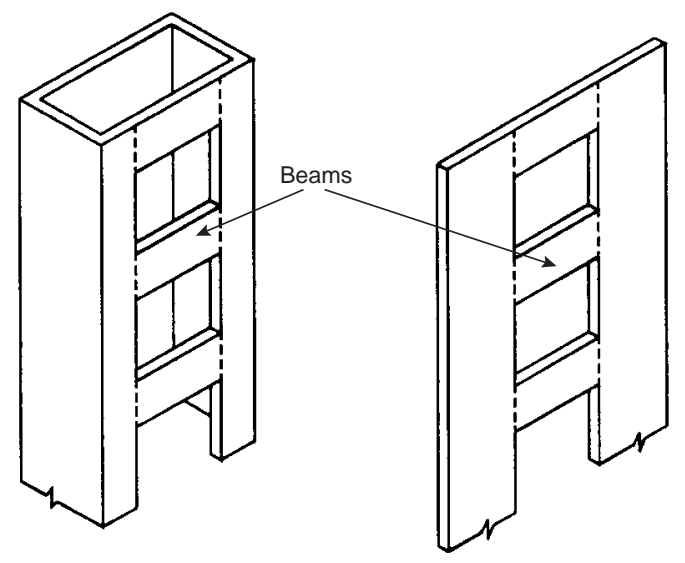

(a)

(b)

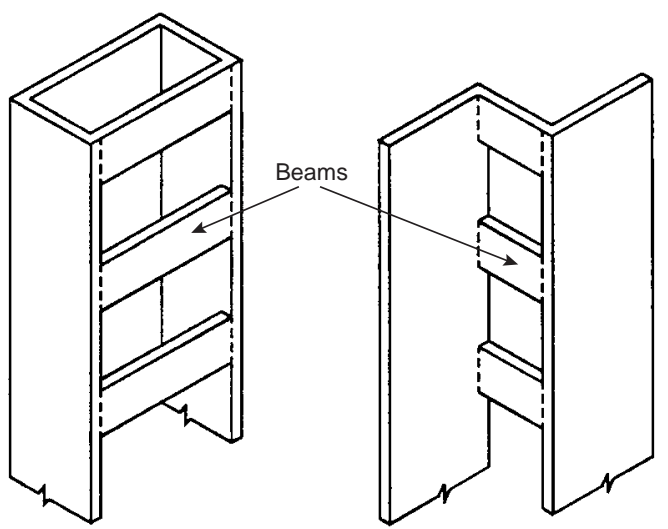

(a)

(b)

Fig. 2. Non-planar coupling beams
Fig. 1. Coplanar coupling beams
5. In the present study, the stress concentration and local deformation at non-planar beam-wall joints were analysed using the finite-element method. A parametric study of the combined influences of the storey height, wall width, wall thickness, beam length, beam breadth, beam depth, etc. was carried out. On the basis of the results of the parametric study, a simple structural model of the non-planar beam-wall joint has been developed. With this model, the coupling effect of the non-planar coupling beams can be easily incorporated in shear/core wall analysis and the concentrated out-of-plane bending moment can be allowed for in reinforcement detailing. This model is particularly suitable for computer analysis of shear/ core wall systems using the frame method or finite-element method.

\section{Parametric study by finite-element analysis}

6. A parametric study of the local deformation around non-planar beam-wall joints was carried out by finite-element analysis. The general layout of the structural models analysed is shown in Fig. 3. Each of the models studied represents a portion of a core wall 
partially closed by non-planar coupling beams, as shown in Fig. 3(a). These models are basically similar to the core wall models investigated by Roberts and Achour. ${ }^{7}$ Following Roberts and Achour's practice, it is assumed that the points of contraflexure of the out-of-plane bending of the side walls are at the midheight of each storey, while those of the coupling beams are at their midspan locations. From the core wall structure, one side wall spanning a storey together with a coupling beam connected to it is cut out along their points of contraflexure for detailed analysis. Fig. 3(b) depicts the cut-out portion analysed.

7. The software used for the finite-element analysis was a commercially available program called SAP90. ${ }^{9}$ A quadrilateral shell element selected from the element library of SAP90 was used to model both the wall and the beam. The element chosen was a four-node thin shell element called SHELL, which, according to the user's manual, is actually a combination of a membrane element and a plate-bending element. Each node of the element has six degrees of freedom, three of which are translational and the other three rotational.

8. The mesh used for the finite-element analysis is shown in Fig. 4. In order to better capture the high stress gradient around the beam-wall joint, a higher density of finite element was employed for the parts of the beam and the wall near the joint. The numbers of elements used to model the beam and the wall were $30 \times 16$ and $48 \times 40$, respectively. Added together, a total of 2400 shell elements was used for the analysis. In order to allow for the finite breadth of the beam, the translational displacements of the wall within the beam-wall interface area (the area bounded by thick lines in Fig. 4) were forced to be uniform across the breadth of the joint by applying the masterslave technique to each row of nodes on the same horizontal line within the interface area. Regarding the boundary conditions, the upper and lower edges of the wall, which were actually the lines of contraflexure of the outof-plane bending of the wall, were treated as hinge supports. The vertical edge of the wall connected to another wall panel of the core wall was also treated as a hinge support. All these three edges were allowed to rotate freely but restrained from any translational movement. The remaining edges were all assumed to be free in both translation and rotation.

9. A convergence study in which several different mesh finenesses were tried for the analysis and the corresponding numerical results for the beam tip deflection were compared was carried out before the main parametric study to investigate the effect of the mesh fineness on the accuracy of the numerical

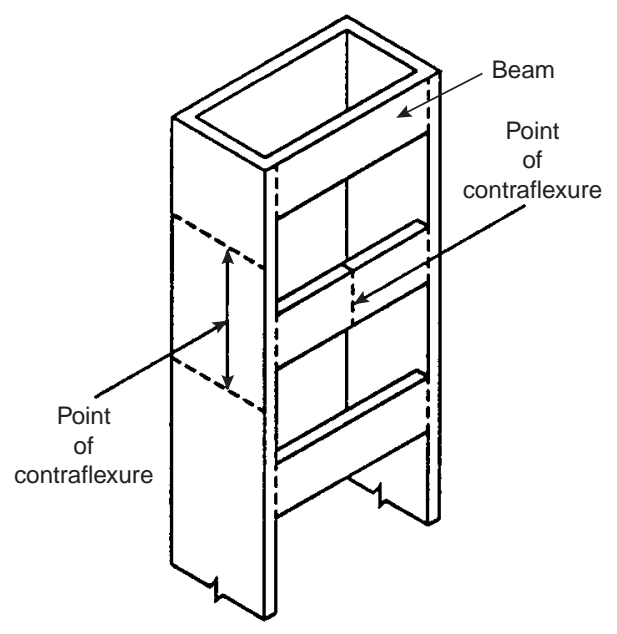

(a)

results. It was found that a further increase of the number of elements to four times the original number by reducing the element size by one-half produced less than $2 \%$ difference in the beam tip deflection. Hence, it may be concluded that the mesh size adopted here was sufficiently fine for the purpose of most practical applications.

10. The structural parameters investigated were the storey height $H$, wall width $W$, wall thickness $T$, beam length $L$, beam breadth $B$ and beam depth $D$. Since the six parameters can be combined in many different ways to yield a very large number of combinations, it is not quite feasible to analyse all possible combinations of them. In order to deal with this difficulty, the parametric study was conducted in three stages, each consisting of one series of model analyses. The three series of model analyses are listed below:

(a) series 1: H, T and $B$ were kept constant while $W, L$ and $D$ were taken as variables

(b) series 2: $W, L, B$ and $D$ were kept constant while $H$ and $T$ were taken as variables

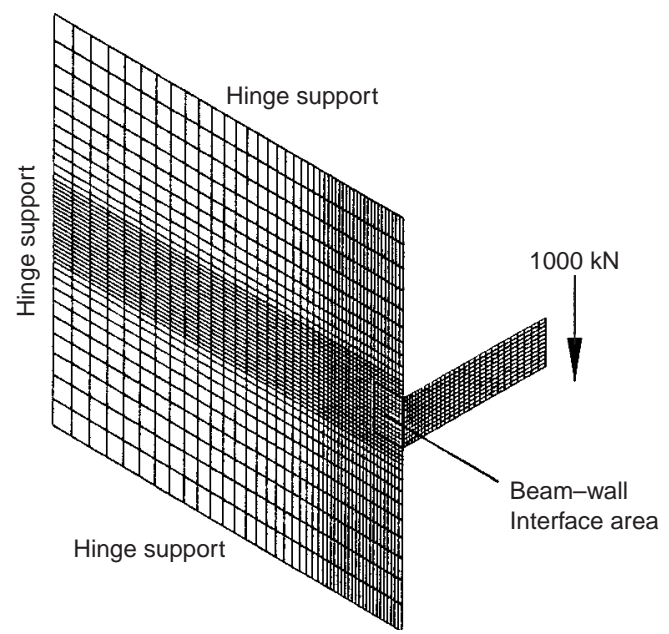

Fig. 3. General layout of structural models analysed
Fig. 4. Finite-element mesh used 
(c) series 3: $W, T, L$ and $D$ were kept constant while $H$ and $B$ were taken as variables.

In each series, the number of variables to be studied was kept at not more than three so that the total number of models that needed to be analysed was more manageable.

11. The material properties were assumed to have the following values throughout the entire study:

(a) modulus of elasticity $E=24000 \mathrm{~N} / \mathrm{mm}^{2}$

(b) shear modulus $G=10000 \mathrm{~N} / \mathrm{mm}^{2}$

(c) Poisson's ratio $v=0 \cdot 20$.

These are typical values for the concrete normally used for the construction of shear/ core walls. The applied load in each analysis consisted of a vertical load of $1000 \mathrm{kN}$ acting downward at the beam tip.

12. Each finite-element analysis produced a fair volume of numerical results and thus the total amount of data that needed to be studied was quite large. Fortunately, all the models analysed were found to behave in a similar way. A typical deformed shape of the model is shown in Fig. 5. From the shape of the deflection of the wall and the bending moment distribution in it, it can be seen that, generally speaking, only a vertical strip of the wall near the beam-wall joint is significantly deflected or bent out of plane when a vertical load is applied at the tip of the beam. In other words, only a vertical strip of the wall near the joint is really effective in resisting the out-of-plane bending moment induced by the vertical load acting on the non-planar coupling beam; the remaining part of the wall does not contribute much to the rotational stiffness of the joint. On the basis of this observation, an equivalent-frame model for simulating the behaviour of non-planar beamwall joints was proposed and is described in the next section.

\section{Proposed structural model for non- planar beam-wall joints}

13. An equivalent-frame model to simulate the structural behaviour of non-planar beamwall joints is described here. As illustrated in Fig. 6, the equivalent frame consists of a fictitious column and a fictitious beam. The fictitious column is assumed to have the same thickness as the wall and an effective width which is to be determined by the parametric study. In order to allow for the finite depth of the coupling beam, the portion of the fictitious column which coincides with the depth of the beam is treated as a vertical rigid arm. Likewise, the fictitious beam is assumed to have the same thickness as the wall and an effective depth to be determined. When the coupling beam connected to the non-planar beam-wall joint is subjected to a vertical shear load, the beam-wall joint rotates, thereby causing

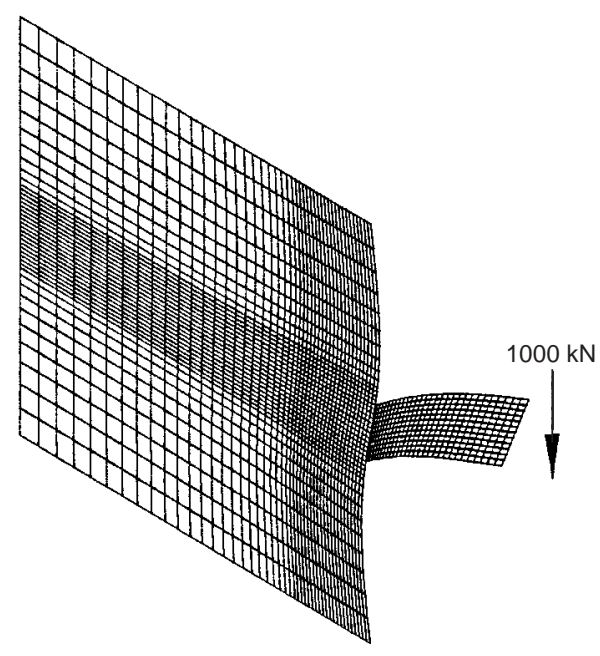

Fig. 5. Typical deformed shape of the models analysed

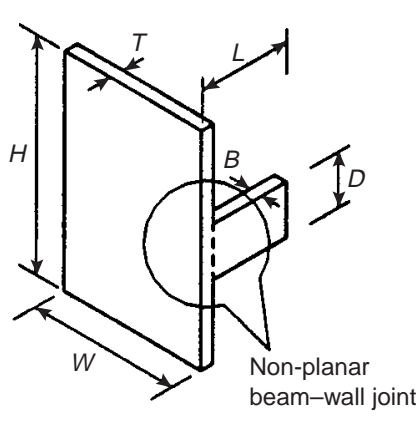

(a)

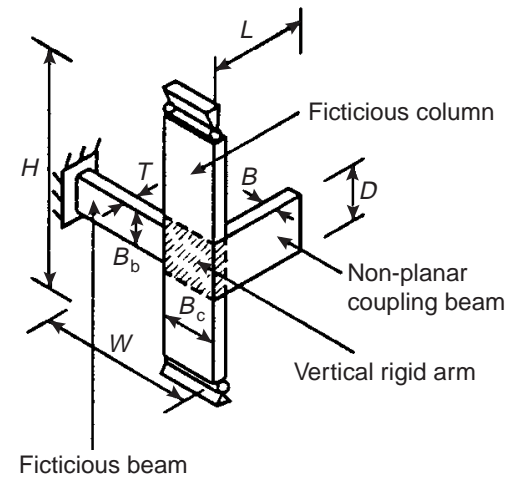

(b) bending of the fictitious column and twisting of the fictitious beam. The rotational stiffness of the non-planar beam-wall joint may be evaluated as

$$
K_{\theta}=\frac{12 E I H^{2}}{(H-D)^{3}}+\frac{G J}{W}
$$

in which $I$ is the moment of inertia of the fictitious column and $J$ is the torsional inertia of the fictitious beam. The values of $I$ and $J$ are given by

$$
\begin{aligned}
I & =\frac{1}{12} B_{\mathrm{c}} T^{3} \\
J & =\frac{1}{3} B_{\mathrm{b}} T^{3}
\end{aligned}
$$

where $B_{\mathrm{c}}$ and $B_{\mathrm{b}}$ are the effective width of the fictitious column and the effective depth of the fictitious beam, respectively. Substituting these values of $I$ and $J$ into equation (1), the rotational stiffness of the joint is obtained as

$$
K_{\theta}=E T^{3} \frac{B_{\mathrm{c}} H^{2}}{(H-D)^{3}}+G T^{3} \frac{B_{\mathrm{b}}}{3 W}
$$

\section{Results of parametric study}

14. In the first series of model analyses (series 1 ), the storey height $H$, wall thickness $T$
Fig. 6. Proposed equivalent-frame model for non-planar beam-wall joint 
Table 1. Series 1 results-beam tip deflection: $\mathrm{mm}$

\begin{tabular}{|c|c|c|c|c|c|c|}
\hline \multirow{2}{*}{$\begin{array}{c}\text { Beam length: } \\
\mathrm{mm}\end{array}$} & \multirow{2}{*}{$\begin{array}{l}\text { Beam depth: } \\
\mathrm{mm}\end{array}$} & \multicolumn{5}{|c|}{ Wall width: $\mathrm{mm}$} \\
\hline & & 2000 & 4000 & 6000 & 8000 & 10000 \\
\hline 1000 & $\begin{array}{r}400 \\
600 \\
800 \\
1000\end{array}$ & $\begin{array}{r}14 \cdot 367 \\
7 \cdot 196 \\
4 \cdot 936 \\
3 \cdot 796\end{array}$ & $\begin{array}{r}14 \cdot 493 \\
7 \cdot 323 \\
5 \cdot 061 \\
3 \cdot 917\end{array}$ & $\begin{array}{r}14 \cdot 503 \\
7 \cdot 332 \\
5 \cdot 070 \\
3.925\end{array}$ & $\begin{array}{r}14 \cdot 505 \\
7 \cdot 334 \\
5 \cdot 072 \\
3 \cdot 926\end{array}$ & $\begin{array}{r}14.505 \\
7 \cdot 334 \\
5 \cdot 072 \\
3 \cdot 926\end{array}$ \\
\hline 2000 & $\begin{array}{r}400 \\
600 \\
800 \\
1000\end{array}$ & $\begin{array}{l}89 \cdot 770 \\
37 \cdot 345 \\
22 \cdot 731 \\
16 \cdot 288\end{array}$ & $\begin{array}{l}90 \cdot 127 \\
37 \cdot 712 \\
23 \cdot 098 \\
16 \cdot 645\end{array}$ & $\begin{array}{l}90 \cdot 137 \\
37 \cdot 722 \\
23 \cdot 107 \\
16 \cdot 654\end{array}$ & $\begin{array}{l}90 \cdot 140 \\
37 \cdot 724 \\
23 \cdot 109 \\
16 \cdot 656\end{array}$ & $\begin{array}{l}90 \cdot 140 \\
37 \cdot 724 \\
23 \cdot 109 \\
16 \cdot 656\end{array}$ \\
\hline
\end{tabular}

and beam breadth $B$ were kept constant and given the following values: $H=4000 \mathrm{~mm}$, $T=300 \mathrm{~mm}$ and $B=300 \mathrm{~mm}$. The wall width $W$, beam length $L$ and beam depth $D$ were each assigned one of the following values in turn: $W=2000,4000,6000,8000,10000 \mathrm{~mm}$; $L=1000,2000 \mathrm{~mm} ; D=400,600,800,1000 \mathrm{~mm}$. Altogether, forty combinations of the above three parameters were analysed.

15. The series 1 results for the beam tip deflection are tabulated in Table 1 . The deflection at the beam tip of each model is due partly to the rotation of the beam-wall joint, partly to the vertical displacement of the joint and partly to the flexural and shear deformations of the beam. Deducting the part due to the flexural and shear deformations of the beam and the part due to the vertical displacement of the joint from the beam tip deflection, and dividing the remaining beam tip deflection by the beam length, the joint rotation in each model can be obtained. From the joint rotation so evaluated, the rotational stiffness of the joint may be determined as the ratio of the bending moment acting at the joint to the resulting joint rotation. Table 2 presents the rotational stiffnesses of the beam-wall joints so derived.

16. It can be seen from Table 2 that the beam length has basically no effect on the rotational stiffness of the non-planar beam-wall joint. This is expected because the rotational stiffness of the joint depends solely on the out- of-plane bending stiffness of the wall and the length of the beam should have no effect on the wall stiffness. On the other hand, the rotational stiffness of the joint increases significantly with the beam depth. This can be explained by the fact that the part of the wall within the beam-wall interface area is stiffened by the beam connected to it and thus a greater beam depth would lead to a greater rotational stiffness.

17. It can also be seen that the rotational stiffness of the joint varies slightly with the wall width, being slightly higher when the wall width is small compared with the storey height and more or less equal to a constant value when the wall width is greater than the storey height. This indicates that the boundary restraint at the vertical wall edge opposite to the beamwall joint has little influence on the rotational stiffness of the joint when the vertical wall edge is at a distance greater than the storey height away from the joint. The effect of the wall width on the rotational stiffness of the joint is simulated by the fictitious beam in the proposed equivalent-frame model, which has a length equal to the wall width and is subjected to twisting when the joint rotates. For given structural parameters, the corresponding value of the effective depth of the fictitious beam $B_{\mathrm{b}}$ may be evaluated by assuming that the rotational stiffness is related to the wall width by equation (4) and applying regression analysis to

Table 2. Series 1 results—rotational stiffness of the joint: $10^{11} \mathrm{Nmm} /$ radian

\begin{tabular}{c|c|c|c|c|c|c}
\hline \multirow{2}{*}{$\begin{array}{c}\text { Beam length: } \\
\mathrm{mm}\end{array}$} & $\begin{array}{c}\text { Beam depth: } \\
\mathrm{mm}\end{array}$ & \multicolumn{5}{|c}{ Wall width: mm } \\
\cline { 3 - 6 } & & 2000 & 4000 & 6000 & 8000 & 10000 \\
\hline \multirow{3}{*}{1000} & 400 & $2 \cdot 207$ & $2 \cdot 147$ & $2 \cdot 142$ & $2 \cdot 141$ & $2 \cdot 141$ \\
& 600 & $2 \cdot 630$ & $2 \cdot 545$ & $2 \cdot 539$ & $2 \cdot 537$ & $2 \cdot 537$ \\
& 800 & $3 \cdot 128$ & $3 \cdot 010$ & $3 \cdot 002$ & $3 \cdot 000$ & $3 \cdot 000$ \\
& 1000 & $3 \cdot 723$ & $3 \cdot 563$ & $3 \cdot 552$ & $3 \cdot 551$ & $3 \cdot 551$ \\
\hline \multirow{3}{*}{2000} & 400 & $2 \cdot 201$ & $2 \cdot 159$ & $2 \cdot 157$ & $2 \cdot 157$ & $2 \cdot 157$ \\
& 600 & $2 \cdot 617$ & $2 \cdot 556$ & $2 \cdot 554$ & $2 \cdot 554$ & $2 \cdot 554$ \\
& 800 & $3 \cdot 102$ & $3 \cdot 016$ & $3 \cdot 014$ & $3 \cdot 013$ & $3 \cdot 013$ \\
& 1000 & $3 \cdot 673$ & $3 \cdot 556$ & $3 \cdot 554$ & $3 \cdot 553$ & $3 \cdot 553$ \\
\hline
\end{tabular}


Table 3. Series 1 results - effective width of fictitious column: $m m$

\begin{tabular}{c|c|c|c|c|c|c}
\hline \multirow{2}{*}{$\begin{array}{c}\text { Beam length: } \\
\text { mm }\end{array}$} & $\begin{array}{c}\text { Beam depth: } \\
\text { mm }\end{array}$ & \multicolumn{5}{|c}{ Wall width: mm } \\
\cline { 3 - 7 } & & 2000 & 4000 & 6000 & 8000 & 10000 \\
\hline \multirow{3}{*}{1000} & 400 & 939 & 939 & 946 & 950 & 953 \\
& 600 & 952 & 942 & 947 & 939 & 953 \\
& 800 & 951 & 932 & 936 & 917 & 918 \\
\hline \multirow{3}{*}{2000} & 1000 & 938 & 912 & 915 & 957 & 960 \\
& 400 & 936 & 945 & 953 & 957 & 959 \\
& 600 & 947 & 946 & 953 & 943 & 945 \\
& 800 & 942 & 934 & 940 & 917 & 919 \\
\hline
\end{tabular}

the set of rotational stiffness results for the five different values of wall width presented in Table 2. The regression analysis revealed that, within the ranges of structural parameters investigated in this particular series of model analyses, the value of $B_{\mathrm{b}}$ changes slightly with the beam depth but is generally quite close to $270 \mathrm{~mm}$.

18. Neglecting the small variation of $B_{\mathrm{b}}$ with the beam depth and assuming that $B_{\mathrm{b}}$ has a constant value of $270 \mathrm{~mm}$, the effective width of the fictitious column $B_{\mathrm{c}}$ may be evaluated by solving equation (4) for each set of structural parameters. The values of $B_{\mathrm{c}}$ so determined are tabulated in Table 3 . It is found that although the rotational stiffness of the joint increases with the beam depth, the effective width of the fictitious column is virtually independent of the beam depth. In the equivalent-frame model, the stiffening effect of the coupling beam on the part of the wall within the beam-wall interface area is already allowed for by the vertical rigid arm in the fictitious column and thus no adjustment of the effective width of the fictitious column is necessary to account for the effect of the beam depth. The results presented in Table 3 also show that the variation of $B_{\mathrm{c}}$ with the wall width is very small and hence the value of $B_{\mathrm{c}}$ may be taken as independent of the wall width.

19. In the second series of model analyses (series 2), the wall width $W$, beam length $L$, beam breadth $B$ and beam depth $D$ were kept constant and given the following values: $W=8000 \mathrm{~mm}, L=2000 \mathrm{~mm}, B=300 \mathrm{~mm}$, $D=600 \mathrm{~mm}$. The storey height $H$ and wall thickness $T$ were each assigned one of the following values in turn: $H=3000,4000,5000$, $6000 \mathrm{~mm} ; T=200,300,400 \mathrm{~mm}$. Altogether, twelve combinations of the above two parameters were analysed.

20. Following the same procedure as in the series 1 analyses, it was found that the effective depth of the fictitious beam $B_{\mathrm{b}}$ increased with the storey height $H$ and was in general approximately equal to $H / 15$. Assuming that the effective depth of the fictitious beam is related to the storey height by the equation $B_{\mathrm{b}}=H / 15$, the corresponding results for the effective width of the fictitious column were evaluated and are listed in Table 4 . From these results, it is evident that the wall thickness has no effect on the effective width of the fictitious column. The wall thickness does have an important effect on the rotational stiffness of the joint but its effect is already taken into account by the term $T^{3}$ in equation (4). Since the values of both $B_{\mathrm{b}}$ and $B_{\mathrm{c}}$ in equation (4) are independent of the wall thickness, it may be said that the rotational stiffness of the joint is proportional to the cube of the wall thickness $T$. From the results presented in Table 4 , it is also evident that the effective width of the fictitious column $B_{\text {c }}$ increases with the storey height $H$.

Comparison of the corresponding results for the effective width of the fictitious column at different storey height indicates that $B_{\mathrm{c}}$ is more or less a linear function of $H$.

21. At this stage, it becomes clear that the effective width of the fictitious column $B_{\mathrm{c}}$ is not dependent on the beam length, beam depth, wall width or wall thickness. The major structural parameter that determines the value of $B_{\mathrm{c}}$ appears to be the storey height. Another para-

Table 4. Series 2 results - effective width of fictitious column: $\mathrm{mm}$

\begin{tabular}{c|c|c|c|c}
\hline \multirow{2}{*}{$\begin{array}{c}\text { Wall thickness: } \\
\text { mm }\end{array}$} & 3000 & 4000 & 5000 & 6000 \\
\cline { 2 - 5 } & 778 & 968 & 1142 & 1305 \\
200 & 775 & 957 & 1130 & 1295 \\
300 & 772 & 965 & 1140 & 1297 \\
\hline
\end{tabular}


meter that could affect the value of $B_{\mathrm{c}}$ is the beam breadth, which has not been considered so far. In order to study the combined effects of the storey height and beam breadth, they were treated as variables in the next series of analyses.

22. In the third series of model analyses (series 3 ), the wall width $W$, wall thickness $T$, beam length $L$ and beam depth $D$ were kept constant, as follows: $W=8000 \mathrm{~mm}$, $T=300 \mathrm{~mm}, L=2000 \mathrm{~mm}, D=600 \mathrm{~mm}$. The storey height $H$ and beam breadth $B$ were each assigned one of the following values in turn: $H=3000,4000,5000,6000 \mathrm{~mm} ; B=200,300$, $400,600 \mathrm{~mm}$. Sixteen combinations of the above two parameters were analysed. The corresponding results for the effective width of the fictitious column are presented in Table 5.

23. It is obvious from Table 5 that the effective width of the fictitious column increases with both the beam breadth and the storey height. Close inspection of the variation of the effective width of the fictitious column $B_{\mathrm{c}}$ with the beam breadth $B$ indicates that $B_{\mathrm{c}}$ is roughly a linear function of $B$. Since $B_{\mathrm{c}}$ is also a linear function of the storey height $H$, it is quite possible that $B_{\mathrm{c}}$ is related to $B$ and $H$ by the following equation:

$$
B_{\mathrm{c}}=\alpha \mathrm{B}+\beta \mathrm{H}
$$

in which $\alpha$ and $\beta$ are constants of proportionality. Assuming the above equation and applying regression analysis to the set of $B_{\mathrm{c}}$ values in Table 5 , the values of $\alpha$ and $\beta$ were obtained as 0.95 and $0 \cdot 17$, respectively. Hence, the effective width of the fictitious column may be estimated from

$$
B_{\mathrm{c}}=0 \cdot 95 B+0 \cdot 17 H
$$

However, intuitively, a value of 1.0 is more appropriate for $\alpha$ as the strip of the wall directly connected to the beam at the beamwall interface and having a width equal to $B$ should be fully effective in resisting the out-ofplane moment applied at the joint. Therefore, the above equation should be modified to

$$
B_{\mathrm{c}}=B+0 \cdot 17 H
$$

Comparison of the values of $B_{\mathrm{c}}$ predicted by the above equation with the corresponding values presented in Table 5 shows that the equation is accurate to within a $5 \%$ error.
24. Using the above values of $B_{\mathrm{b}}$ and $B_{\mathrm{c}}$, it can be shown that, provided the wall width is greater than half of the storey height, the contribution of the fictitious beam to the rotational stiffness of the beam-wall joint is generally less than $5 \%$. Since this condition is satisfied in most practical cases, the fictitious beam may actually be neglected and the equivalent-frame model simplified to just a fictitious column.

\section{Comparison with equivalent-shear- diaphragm model}

25. The equivalent-shear-diaphragm model proposed by Roberts and Achour, ${ }^{7}$ which is based on an assumed bending shape of the side walls, gives the following equation for the rotational stiffness of the beam-wall joint:

$$
\begin{aligned}
& K_{\theta}=\frac{E T^{3}}{12\left(1-v^{2}\right)} \\
& \times\left(2(1-v) \frac{D}{W}+\frac{2 \pi^{2} W H^{2}}{3(H-D)^{3}}+(1-v) \frac{\left(H^{2}+2 D^{2}\right)}{(H-D) W}\right)
\end{aligned}
$$

After rearrangement, the above equation becomes

$$
\begin{aligned}
K_{\theta}= & E T^{3} \frac{\left\{\pi^{2} /\left[18\left(1-v^{2}\right)\right]\right\} W H^{2}}{(H-D)^{3}} \\
& +G T^{3} \frac{\{H(H+2 D) /[2(H-D)]\}}{3 W}
\end{aligned}
$$

Comparing this equation with equation (4), it can be seen that the two equations are of similar form and that the rotational stiffness of the beam-wall joint derived by Roberts and Achour's equivalent-shear-diaphragm model may also be expressed in the form of equation (4) with $B_{\mathrm{b}}$ and $B_{\mathrm{c}}$ assigned the following values:

$$
B_{\mathrm{b}}=\frac{H(H+2 D)}{2(H-D)} \approx 0.50 H \quad \text { if } D / H \text { is small }
$$

$$
B_{\mathrm{c}}=\frac{\pi^{2}}{18\left(1-v^{2}\right)} W=0.57 W \quad \text { if } v=0.20
$$

At this stage, the following discrepancies are obvious: $(a)$ the equivalent-shear-diaphragm model yields a somewhat larger value of

Table 5. Series 3 results-effective width of fictitious column: $\mathrm{mm}$

\begin{tabular}{c|r|r|r|r}
\hline \multirow{2}{*}{$\begin{array}{c}\text { Beam breadth: } \\
\mathrm{mm}\end{array}$} & 3000 & 4000 & 5000 & \multicolumn{5}{|c}{ Storey height: $\mathrm{mm}$} \\
\cline { 2 - 5 } & 688 & 866 & 1044 & 6000 \\
\hline 200 & 775 & 957 & 1130 & 1201 \\
300 & 868 & 1061 & 1247 & 1295 \\
400 & 1049 & 1256 & 1441 & 1409 \\
600 & & & 1599 \\
\hline
\end{tabular}




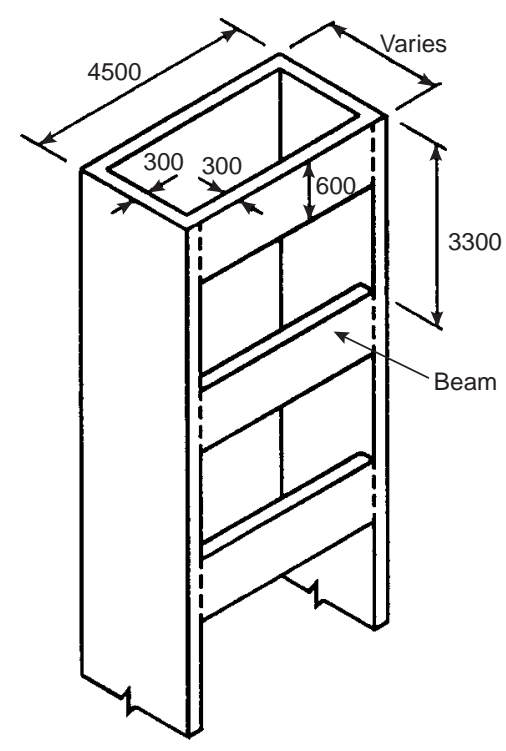

(a)

$B_{\mathrm{b}}$ compared with that obtained by finiteelement analysis in the present study; $(b)$ the equivalent-shear-diaphragm model yields a value of $B_{\mathrm{c}}$ which increases with the wall width $W$, while the finite-element results in the present study produce a value that is independent of $W$; and $(c)$ the rotational stiffness obtained by the shear diaphragm method is generally higher than that obtained in the present study, especially when the wall width is large compared with the storey height. The main reason for these discrepancies is the occurrence of stress concentration near the beam-wall joints, which is not easy to deal with by using an assumed bending shape of the walls. As stress concentration around the beam-wall joints has not been properly allowed for, the equivalent-shear-diaphragm method tends to overestimate the rotational stiffness of non-planar beam-wall joints.

26. Although the discrepancy in joint stiffness between the equivalent-shear-diaphragm model and the equivalent-frame model proposed here is quite large, the actual difference in the effective shear stiffness of the non-planar coupling beam, which is defined as the ratio of the shear load in the coupling beam to the relative shear displacement of the two ends of the beam, is not that large. Consider a typical core wall with non-planar coupling beams whose layout and dimensions are as shown in Fig. 7(a). All dimensions of the core wall are kept constant here, except the width of the side walls, which is varied from $1500 \mathrm{~mm}$ to $4500 \mathrm{~mm}$ in steps of $500 \mathrm{~mm}$. The values of beam effective shear stiffness obtained by the two different models are plotted in Fig. 7(b). It is seen that for the particular model analysed, the difference in effective beam stiffness is of the order of $11 \%$ when the wall width is

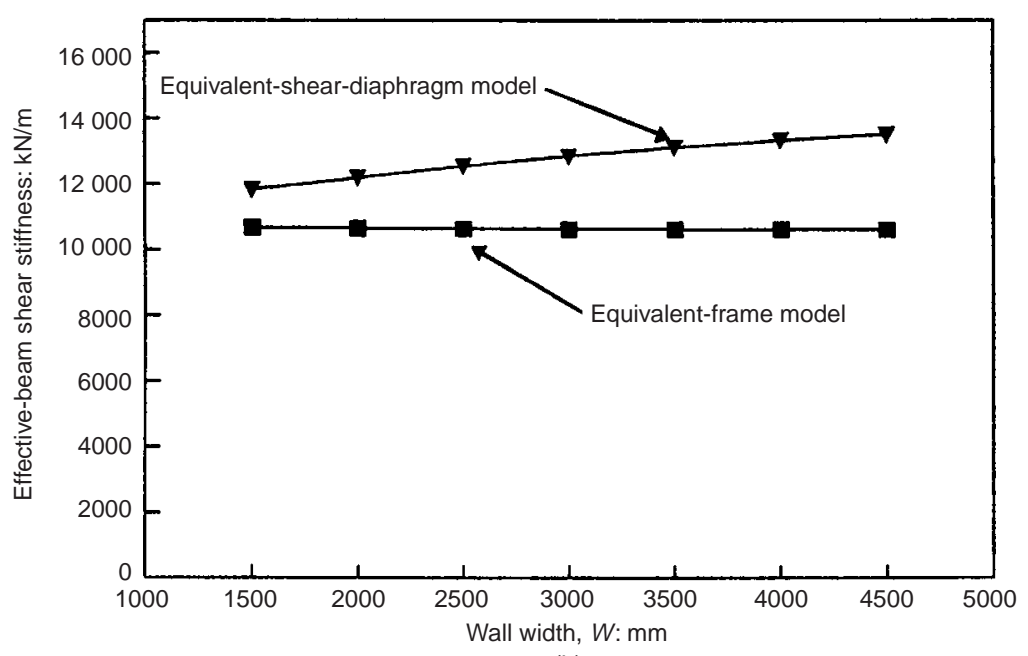

(b)

Fig. 7. Comparison of equivalent-frame model with equivalent-sheardiaphragm model (dimensions in $\mathrm{mm}$ )

relatively small but becomes as large as $27 \%$ when the wall width is relatively large.

\section{Numerical examples}

\section{Example 1-core wall with non-planar coupling beams}

27. In order to illustrate the application of the proposed equivalent-frame model and study the possible coupling effect of non-planar coupling beams, a typical core wall partially closed by non-planar coupling beams was analysed. The core wall studied is shown in Fig. 8(a). It was subjected to a torsion of $100 \mathrm{kN} \mathrm{m}$ at the top of the core wall. The core wall was first analysed with the non-planar beam-wall joints treated as hinges (the current practice adopted by most design engineers) so that in effect the coupling effect of the beams was neglected, and the wall was then reanalyzed with the coupling effect of the beams allowed for using the proposed equivalentframe model. In the numerical analysis, the inplane actions of the wall panels were modelled by plane stress elements, while the out-of-plane bending actions of the wall panels were modelled by the equivalent-frame model. As the fictitious beam in the equivalent-frame model was not expected to contribute more than $5 \%$ to the rotational stiffness of the beam-wall joint, it was discarded and the equivalent-frame model was simplified to just a fictitious column. Fig. 8(b) presents the results of the analysis for the torsional rotations of the core wall over the height of the wall structure. Comparing the results for the wall structure with the coupling effect of the beams neglected with the corresponding results with the coupling effects allowed for, it is obvious that the coupling effect of the non-planar coupling beams can 


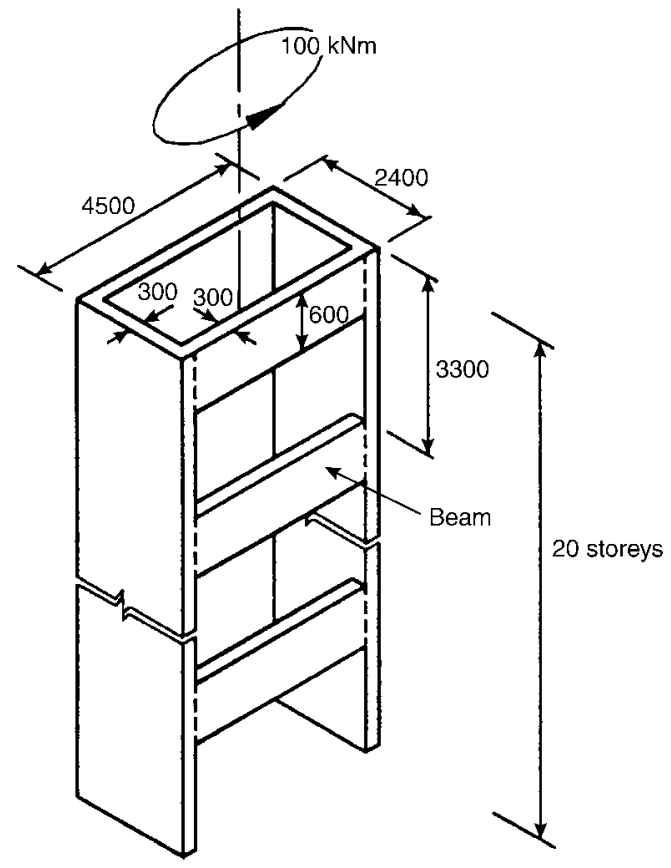

(a)

substantially increase the torsional stiffness of a core wall structure. Neglect of the coupling effect of non-planar coupling beams could lead to significant errors in the torsional rigidity of buildings with such a kind of core walls inside.

Example 2-shear walls coupled by non-planar coupling beams

28. Another example, shown in Fig. 9(a), is a structure consisting of two pairs of shear walls coupled by beams which lie in a vertical plane perpendicular to the walls. This type of

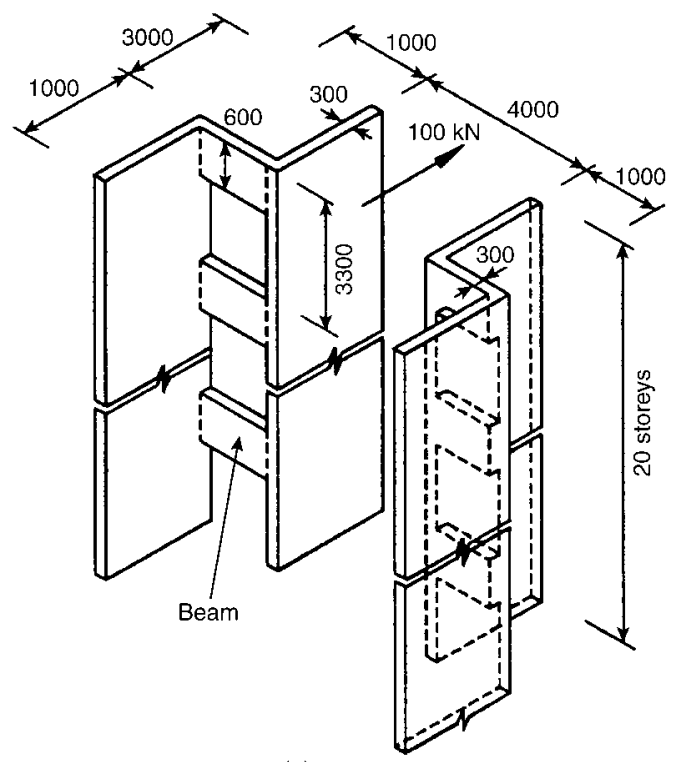

(a)

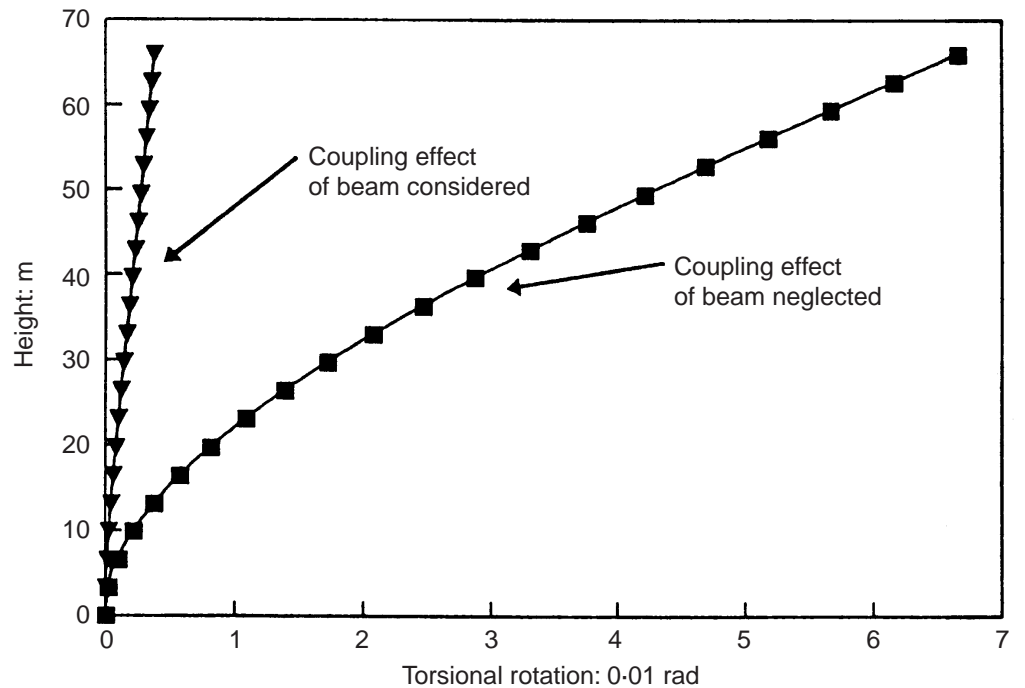

(b)

Fig. 8. Example 1-torsion of core wall with non-planar coupling beams (dimensions in $\mathrm{mm}$ )

non-planar coupled shear wall is quite common when a $Z$-shaped shear wall is pierced with openings in the central wall panel for windows. As before, in order to illustrate the coupling effect of the non-planar coupling beams, the wall structure was first analysed with the nonplanar beam-wall joints treated as hinges so that the coupling effect of the beams was effectively ignored, and then it was reanalysed with the coupling effect of the beams allowed for using the proposed equivalent-frame model. Again, the in-plane actions of the wall panels were modelled by plane stress elements and the

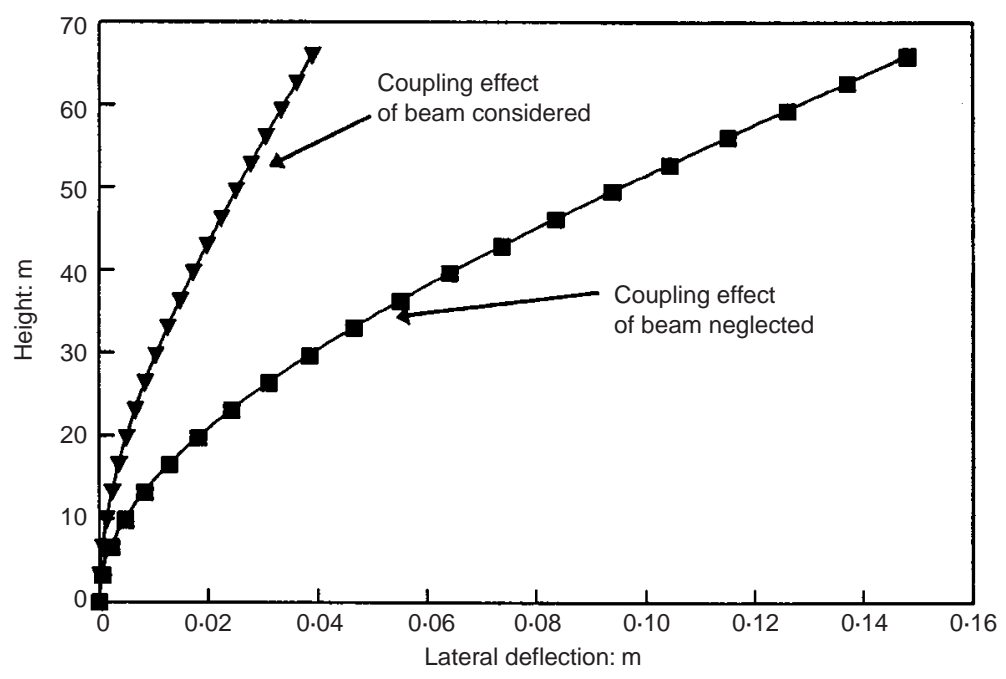

(b)

Fig. 9. Example 2-deflection of shear walls coupled by non-planar coupling beams (dimensions in $\mathrm{mm}$ ) 
out-of-plane bending actions by the equivalentframe model. As the vertical wall edges opposite to the beam-wall joints are not connected to any other wall panels, the fictitious beam in the equivalent frame was discarded and the outof-plane bending actions of the walls were modelled by fictitious columns only. The numerical results for the lateral deflections of the structure are plotted in Fig. 9(b), where it can be seen that the coupling effect of the nonplanar coupling beams can reduce the lateral deflection of such a non-planar coupled wall structure by more than $70 \%$.

\section{Reinforcement detailing for non-planar beam-wall joints}

29. The out-of-plane bending moment acting on the wall at the beam-wall joint from the coupling beam is resisted partly by torsion of the fictitious beam and partly by bending of the fictitious column. However, since the proportion of rotational stiffness of the joint contributed by the torsional stiffness of the fictitious beam is generally less than $5 \%$, the torsional resistance of the fictitious beam may be neglected and the bending moment from the coupling beam may be assumed to be taken entirely by the fictitious column. In other words, it may be assumed that the bending moment acting from the coupling beam is resisted solely by vertical bending of the wall.

30. Owing to stress concentration near the beam-wall joint, the vertical bending moment (the bending moment which causes vertical stresses) in the wall is far from being uniformly distributed in the horizontal direction. In general, the vertical bending moment in the wall is highest at the joint and decreases fairly rapidly with the horizontal distance from the joint. Studying the numerical results of the models analysed one by one, it has been found that, generally speaking, more than $70 \%$ of the bending moment acting from the coupling beam is resisted by the part of the wall within the effective width of the fictitious column. For simplicity, therefore, it may be assumed that the whole of the bending moment acting from the coupling beam is resisted by a vertical strip of the wall having a width equal to the effective width $B_{\mathrm{c}}$ of the fictitious column.

31. Regarding reinforcement detailing, the vertical strip of the wall having a width equal to the effective width of the fictitious column, which is assumed to carry all the bending moment acting from the coupling beam, should be designed and detailed as a column, taking into account the bending moment acting on it in accordance with the relevant code of practice. On the other hand, when detailing the reinforcement for the coupling beam, the bending moment induced at the ends of the beam due to beam-wall interaction should be properly allowed for and the longitudinal reinforcement so provided adequately anchored into the wall.

\section{Conclusions}

32. A parametric study of the structural behaviour of non-planar beam-wall joints has been carried out using finite-element analysis. On the basis of the finite-element results, an equivalent-frame model was developed for modelling the beam-wall interaction at such joints. Simple formulae for evaluating the member sizes of the equivalent frame $\left(B_{\mathrm{b}}=H / 15\right.$, $\left.B_{\mathrm{c}}=B+0 \cdot 17 H\right)$ were derived from the numerical results. Compared with the existing equivalent-shear-diaphragm model, this new model should be more accurate because the outof-plane bending shape of the wall is evaluated as part of the solution instead of just assumed. The application of the model has been illustrated through two examples, one of a core wall with non-planar coupling beams and the other of shear walls coupled by beams perpendicular to them. It has been shown from these examples that the coupling effect of non-planar coupling beams can be very substantial and should not be ignored. Finally, some guidelines for detailed design of non-planar beam-wall joints can be given as follows: $(a)$ the out-ofplane bending moment acting on the wall from the beam may be assumed to be carried entirely by a vertical strip of the wall having a width equal to $B_{\mathrm{c}}$, which should then be designed and detailed as a column, taking into account the bending moment acting on it; $(b)$ when designing the coupling beam, the bending moment induced in it owing to beam-wall interaction should be properly allowed for and the longitudinal rebars so provided adequately anchored into the wall.

\section{References}

1. MacLeod I. A. Lateral stiffness of shear walls with openings. Proceedings, Symposium on Tall Buildings. Pergamon, New York, 1967, 223-252.

2. Coull A. and Choudhury J. R. Stresses and deflections in coupled shear walls. Journal of the American Concrete Institute, 1967, 64, No. 2 , 65-72.

3. Tso W. K. and Biswas J. K. General analysis of nonplanar coupled shear walls. Journal of the Structural Division of the ASCE, 1973, 99, No. ST3, 365-380.

4. Michael D. The effect of local wall deformations on the inelastic interaction of cross walls coupled by beams. Proceedings, Symposium on Tall Buildings. Pergamon, New York, 1967, 253-270.

5. Bнатт P. Effect of beam-shearwall junction deformations on the flexibility of the connecting beams. Building Science, 1973, 8, 149-151.

6. Kwan A. K. H. Local deformation and rotational degrees of freedom at beam-wall joints. Computers \& Structures, 1993, 48, No. 4, 615-625.

7. Roberts T. M. and Achour B. Torsion and bending of braced thin-walled open sections. 
Journal of Structural Engineering, ASCE, 1990, 116, No. 1, 1-12.

8. Roberts T. M. and Yeung K. W. Bending and torsion of doubly symmetric core shear walls. Thin-Walled Structures, 1992, 14, No. 5, 395410.
9. Habibullah A. and Wilson E. L. SAP9O-a Series of Computer Programs for the Finite Element Analysis of Structures: Users Manual. Computers and Structures Inc., Berkeley, CA, 1991.

Please email, fax or post your discussion contributions to the Secretary:

email: Wilson_1@ice.org.uk; fax: + 44 (0)20 7799 1325; or post to Lesley Wilson, Journals Department, Institution of Civil Engineers, 1-7 Great George Street, London SW1P 3AA. 